Bangladesh J. Bot. 48(1): 43-51, 2019 (March)

\title{
EFFECTS OF SOWING TIME ON GROWTH AND YIELD PERFORMANCE OF SIX HIGH YIELDING VARIETIES OF WHEAT (TRITICUM AESTIVUM L.)
}

\author{
AMM Golam AdaM*and Nargis Jahan \\ Department of Botany, University of Dhaka, Dhaka-1000, Bangladesh
}

Keywords: Wheat, Variety, Sowing time, Growth, Yield performance

\begin{abstract}
A field experiment was conducted to evaluate the growth and yield performance of six high yielding varieties of wheat viz., BARI Gom-23, BARI Gom-24, BARI Gom-25, BARI Gom-26, BARI Gom-27 and BARI Gom-28 in four sowing time. BARI Gom-24 sown on November 29 produced tallest plant after the age of 45 days with significant variations. Results also indicated that November 15 sown BARI Gom-25 resulted the highest number of tillers, leaves and total dry matter per plant throughout the growth ages with a few exceptions where dry matter produced at the age of 60, 75 and 90 days were significantly higher. Yield parameters viz., number of grains per plant, dry weight of spike, 1000-grain weight, yield per plant, yield per hectare and harvest index were recorded maximum from BARI Gom-25. On the other hand, seeds sown on November 15 produced significantly higher value in all yield contributing characters except number of effective tillers and number of grains per spike. In case of combined treatments, November 15 sown wheat showed similar trend in majority of yield parameters of BARI Gom-25 where, dry weight of spike, 1000grain weight, yield per plant and yield per hectare were significantly higher than rest of the treatments. Out of six varieties, BARI Gom-25 was the best performed variety. November 15 sown wheat had remarkable effects on yield attributes and yield of most of the varieties but beyond this time yield of wheat reduced significantly.
\end{abstract}

\section{Introduction}

Wheat (Triticum aestivum L.) is the world's largest cereal crop and consumed every day in almost everyone's life. The International Food Policy Research Institute (IFPRI) projections indicate that the world demand for wheat will rise to 775 million metric tons by 2020 , and $60 \%$ in total by 2050 and at the same time, climate change-induced temperature increases are likely to reduce wheat production in developing countries where around $66 \%$ of all wheat is produced (Rosegrant and Agcaoili 2010). In Bangladesh, it is the second major cereal crop after rice, but rice alone is no more capable of providing balanced and nutritious food for the human, poultry and livestock. At present about 1.4 million metric tons of wheat is being produced in Bangladesh against the national demand of 5.6 million metric tons and the country needs to import about 4.5 million metric tons wheat every year (Azad et al. 2017).

Increasing wheat production is a big challenge for Bangladesh. Among the various agronomic techniques, selection of high yielding variety and optimum sowing time is the easiest way to achieve desired yield because different varieties responded differently for the genotypic characters, input requirement, growth process and the prevailing environment during growing season. Optimum time for wheat is very important due to its own definite requirements for temperature and light for emergence, growth and flowering (Dabre et al. 1993). On the other hand, late sowing of wheat is a major problem in the rice wheat areas of Asia (Hobs and Gupta 2002). Delayed sowing suppressed the yield, caused by reduction in the yield contributing traits like number of tillers, number of grains per spike and grain yield (Ansary et al. 1989). After optimum sowing time, production of wheat decreases about $18 \mathrm{~kg}$ per hectare for each day (Saifuzzaman et al. 2010).

*Author for correspondence: <adam_du04@yahoo.com>. Present address: Department of Botany, Jagannath University, Dhaka-1100, Bangladesh. 
Results of several investigations from abroad showed that sowing time had marked effect on growth and yield of different varieties of wheat (Shah and Akmal 2002, Subhan et al. 2004, Akhtar et al. 2006, Abdullah et al. 2007, Shahzad et al. 2007, Ali et al. 2010, Baloch et al. 2010). But, in Bangladesh very little number of reports are available on wheat in relation to cultural method and varietal effect (Rahman et al. 2010) and sowing time (Rashid et al. 2004, Jahan and Adam 2015). Therefore, an attempt was taken for the selection of the best performed variety of wheat in relation to different sowing time.

\section{Materials and Methods}

A field experiment was conducted at the Botanical Garden of the Department of Botany, University of Dhaka during 2014 - 2015 and was laid out in randomized block design with four replications. Experimental field was prepared conventionally where the total area was $98.56 \mathrm{~m}^{2}$ $(17.60 \mathrm{~m} \times 5.60 \mathrm{~m})$. Nitrogen, phosphorus and potassium content of experimental soil were determined by Micro-Kjeldalh's method (Marr and Cresser 1983), ascorbic acid blue colour method (Murphy and Riley 1962) and flame photometer (Jackson 1973), respectively. Soil contains low amount of nitrogen, very high amount of phosphorus and very low amount of potassium. Fertilizers viz., urea, muriate of potash, gypsum and boric acid were applied at doses recommended by Fertilizer Recommendation Guide (2012). Two-thirds of urea and full of the other fertilizers were applied as basal dose during final land preparation. Cow-dung was also mixed homogenously as recommended by Fertilizer Recommendation Guide (2012). The remaining urea was applied immediately after the first irrigation. Seeds of six high yielding varieties of wheat viz., BARI Gom-23( $\left.\mathrm{V}_{1}\right)$, BARI Gom-24( $\left(\mathrm{V}_{2}\right)$, BARI Gom-25( $\left.\mathrm{V}_{3}\right)$, BARI Gom26( $\left(\mathrm{V}_{4}\right)$, BARI Gom-27( $\left.\mathrm{V}_{5}\right)$ and BARI Gom-28( $\left.\mathrm{V}_{6}\right)$ were collected from BARI, Joydebpur, Gazipur and were sterilized with $0.5 \%$ calcium hypochlorite before sowing. Seeds were sown in lines $20 \mathrm{~cm}$ apart maintaining plant to plant distance of $10 \mathrm{~cm}$ at four sowing time viz. November $15\left(\mathrm{~S}_{1}\right)$, November $22\left(\mathrm{~S}_{2}\right)$, November $29\left(\mathrm{~S}_{3}\right)$ and December $04\left(\mathrm{~S}_{4}\right)$. Cultural practices viz., thinning, irrigation, weeding etc. were done following Hand book of agricultural technology (Chowdhury and Hassan 2013).

Data on plant height, number of tillers, number of leaves and total dry matter per plant were recorded at an interval of 15 days from the age of 30 days up to harvest. Yield attributes and yield per plants were calculated after harvest at the age of 108 days. Twelve plants ( 3 plants from each replication) from each treatment were taken to record data on different growth and yield parameters. Data were analyzed statistically and treatment means were compared by LSD test at $5 \%$ level of significance (Steel et al. 1997).

\section{Results and Discussion}

Results presented in Table 1 showed that varietal effect had significant responses on plant height. At the early stage of growth (30 - 45 days), the maximum plant height was recorded from BARI Gom-28 $\left(\mathrm{V}_{6}\right)$ but, after the age of 45 days BARI Gom-24 $\left(\mathrm{V}_{2}\right)$ showed maximum height up to harvest. In case of sowing time, seeds sown on November $29\left(\mathrm{~S}_{3}\right)$ produced tallest plants all over the growth period but, varied significantly up to the age of 60 days only. Results also showed that plant height responded significantly by combined treatments of variety and sowing time. BARI Gom-24 sown on November $29\left(\mathrm{~V}_{2} \mathrm{~S}_{3}\right)$ resulted maximum height from the ages of 60 days up to harvest where, it was significantly different from most of the treatments. The height of the crop is mainly controlled by the genetic makeup of a genotype and it can also be affected by the environmental factors (Shahzad et al. 2007). Baloch et al. (2010) reported that plant height of wheat differed significantly by planting time. 
The economic yield of most of the cereals is determined by the number of tillers. Results revealed that BARI Gom-27 $\left(\mathrm{V}_{5}\right)$ produced the highest number of tillers per plant all over the growth ages except at harvest (Table 2). Results also revealed that seeds sown on November 15 $\left(\mathrm{S}_{1}\right)$ produced the highest number of tillers per plant than rest of the sowing time throughout the growth periods and were significantly higher at the ages of 45 and 60 days and at harvest.

Table 1. Effect of sowing time on plant height of six varieties of wheat at different ages.

\begin{tabular}{|c|c|c|c|c|c|c|}
\hline Treatments & 30 days & 45 days & 60 days & 75 days & 90 days & At harvest \\
\hline \multicolumn{7}{|l|}{ Variety $(\mathrm{V})$} \\
\hline $\mathrm{V}_{1}$ & $32.07 \mathrm{~cd}$ & $43.22 \mathrm{bc}$ & $71.52 \mathrm{~b}$ & $88.02 \mathrm{ab}$ & $89.68 \mathrm{a}$ & $90.04 \mathrm{a}$ \\
\hline $\mathrm{V}_{2}$ & $33.69 \mathrm{~b}-\mathrm{d}$ & $46.79 \mathrm{ab}$ & $79.28 \mathrm{a}$ & $91.44 \mathrm{a}$ & $92.33 \mathrm{a}$ & $92.63 \mathrm{a}$ \\
\hline $\mathrm{V}_{3}$ & $34.22 \mathrm{bc}$ & $45.13 \mathrm{ab}$ & $73.09 \mathrm{~b}$ & $89.80 \mathrm{ab}$ & $91.42 \mathrm{a}$ & $91.68 \mathrm{a}$ \\
\hline $\mathrm{V}_{4}$ & $35.22 \mathrm{ab}$ & $48.39 \mathrm{a}$ & $72.80 \mathrm{~b}$ & $87.45 \mathrm{~b}$ & $88.67 \mathrm{ab}$ & $89.08 \mathrm{ab}$ \\
\hline $\mathrm{V}_{5}$ & $31.60 \mathrm{~d}$ & $39.51 \mathrm{c}$ & $66.89 \mathrm{c}$ & $88.24 \mathrm{ab}$ & $90.33 \mathrm{a}$ & $90.70 \mathrm{a}$ \\
\hline $\mathrm{V}_{6}$ & $36.26 \mathrm{a}$ & $48.51 \mathrm{a}$ & $72.16 \mathrm{~b}$ & $83.53 \mathrm{c}$ & $84.43 b$ & $84.97 \mathrm{~b}$ \\
\hline \multicolumn{7}{|c|}{ Sowing time (S) } \\
\hline $\mathrm{V}_{1}$ & $33.13 \mathrm{bc}$ & $45.48 \mathrm{ab}$ & $72.18 \mathrm{~b}$ & $86.62 \mathrm{a}$ & $87.80 \mathrm{a}$ & $88.08 \mathrm{a}$ \\
\hline $\mathrm{V}_{2}$ & $35.29 \mathrm{ab}$ & $44.56 \mathrm{ab}$ & $68.58 \mathrm{~b}$ & $87.44 \mathrm{a}$ & $89.95 \mathrm{a}$ & $90.23 \mathrm{a}$ \\
\hline $\mathrm{V}_{3}$ & $35.53 \mathrm{a}$ & $48.80 \mathrm{a}$ & $76.96 \mathrm{a}$ & $90.36 \mathrm{a}$ & $91.27 \mathrm{a}$ & $91.70 \mathrm{a}$ \\
\hline $\begin{array}{l}\mathrm{V}_{4} \\
(\mathrm{~V} \times \mathrm{S})\end{array}$ & $31.42 \mathrm{c}$ & $42.18 \mathrm{~b}$ & $72.77 \mathrm{ab}$ & $87.89 \mathrm{a}$ & $88.87 \mathrm{a}$ & $89.40 \mathrm{a}$ \\
\hline $\mathrm{V}_{1} \mathrm{~S}_{1}$ & $31.71 \mathrm{~h}-\mathrm{j}$ & $43.01 \mathrm{f}-\mathrm{i}$ & $68.29 \mathrm{jk}$ & $83.25 \mathrm{ij}$ & $86.24 \mathrm{gh}$ & $86.80 \mathrm{~g}-\mathrm{i}$ \\
\hline $\mathrm{V}_{2} \mathrm{~S}_{1}$ & $30.63 \mathrm{ij}$ & $44.36 \mathrm{e}-\mathrm{i}$ & 75.99 b-e & 87.35 d-h & 88.34 d-h & $88.61 \mathrm{e}-\mathrm{i}$ \\
\hline $\mathrm{V}_{3} \mathrm{~S}_{1}$ & $32.50 \mathrm{~g}-\mathrm{i}$ & $45.80 \mathrm{c}-\mathrm{g}$ & $71.43 \mathrm{e}-\mathrm{k}$ & $87.99 \mathrm{c}-\mathrm{g}$ & $88.65 \mathrm{c}-\mathrm{h}$ & $88.73 \mathrm{~d}-\mathrm{i}$ \\
\hline $\mathrm{V}_{4} \mathrm{~S}_{1}$ & $34.25 \mathrm{~d}-\mathrm{g}$ & $49.61 \mathrm{a}-\mathrm{c}$ & $73.49 \mathrm{c}-\mathrm{g}$ & $86.24 \mathrm{e}-\mathrm{i}$ & $86.70 \mathrm{f}-\mathrm{h}$ & $87.13 \mathrm{~g}-\mathrm{i}$ \\
\hline $\mathrm{V}_{5} \mathrm{~S}_{1}$ & $32.76 \mathrm{f}-\mathrm{i}$ & $41.90 \mathrm{~g}-\mathrm{i}$ & $69.60 \mathrm{~g}-\mathrm{k}$ & 89.66 b-e & $90.73 \mathrm{a}-\mathrm{g}$ & $90.83 \mathrm{a}-\mathrm{g}$ \\
\hline $\mathrm{V}_{6} \mathrm{~S}_{1}$ & $36.93 \mathrm{a}-\mathrm{c}$ & 48.19 a-e & $74.26 \mathrm{c}-\mathrm{f}$ & $85.24 \mathrm{f}-\mathrm{i}$ & $86.18 \mathrm{gh}$ & $86.39 \mathrm{hi}$ \\
\hline $\mathrm{V}_{1} \mathrm{~S}_{2}$ & 33.04 e-h & $42.36 \mathrm{~g}-\mathrm{i}$ & 68.44 i-k & $87.73 \mathrm{~d}-\mathrm{g}$ & $89.54 \mathrm{~b}-\mathrm{g}$ & $89.54 \mathrm{c}-\mathrm{h}$ \\
\hline $\mathrm{V}_{2} \mathrm{~S}_{2}$ & $36.14 \mathrm{a}-\mathrm{d}$ & $47.80 \mathrm{~b}-\mathrm{f}$ & $76.33 \mathrm{~b}-\mathrm{d}$ & $92.71 \mathrm{ab}$ & 92.89 a-d & $93.06 \mathrm{a}-\mathrm{d}$ \\
\hline $\mathrm{V}_{3} \mathrm{~S}_{2}$ & $35.24 \mathrm{~b}-\mathrm{e}$ & $44.49 \mathrm{~d}-\mathrm{i}$ & $70.53 \mathrm{f}-\mathrm{k}$ & 89.70 b-e & $93.81 \mathrm{ab}$ & $94.06 \mathrm{ab}$ \\
\hline $\mathrm{V}_{4} \mathrm{~S}_{2}$ & $36.99 \mathrm{a}-\mathrm{c}$ & $47.40 \mathrm{~b}-\mathrm{f}$ & $67.46 \mathrm{k}$ & 84.56 g-i & $87.44 \mathrm{f}-\mathrm{h}$ & $87.75 \mathrm{f}-\mathrm{i}$ \\
\hline $\mathrm{V}_{5} \mathrm{~S}_{2}$ & $32.73 \mathrm{f}-\mathrm{i}$ & 37. $20 \mathrm{j}-\mathrm{k}$ & 59.931 & $86.19 \mathrm{e}-\mathrm{i}$ & $91.89 \mathrm{a}-\mathrm{e}$ & $92.33 \mathrm{a}-\mathrm{e}$ \\
\hline $\mathrm{V}_{6} \mathrm{~S}_{2}$ & $37.61 \mathrm{a}$ & $48.11 \mathrm{a}-\mathrm{f}$ & $68.83 \mathrm{~h}-\mathrm{k}$ & $83.74 \mathrm{~h}-\mathrm{j}$ & 84.14 h-i & $84.63 \mathrm{ij}$ \\
\hline $\mathrm{V}_{1} \mathrm{~S}_{3}$ & 33.80 e-h & $47.03 \mathrm{~b}-\mathrm{f}$ & $76.34 \mathrm{~b}-\mathrm{d}$ & 90.16 b-d & $91.00 \mathrm{a}-\mathrm{f}$ & $91.60 \mathrm{a}-\mathrm{f}$ \\
\hline $\mathrm{V}_{2} \mathrm{~S}_{3}$ & $34.93 \mathrm{c}-\mathrm{f}$ & $50.88 \mathrm{ab}$ & $84.63 \mathrm{a}$ & $94.00 \mathrm{a}$ & $94.84 \mathrm{a}$ & $94.91 \mathrm{a}$ \\
\hline $\mathrm{V}_{3} \mathrm{~S}_{3}$ & $36.64 \mathrm{a}-\mathrm{c}$ & $48.74 \mathrm{a}-\mathrm{d}$ & $77.14 \mathrm{~b}-\mathrm{d}$ & $92.90 \mathrm{ab}$ & $93.64 \mathrm{ab}$ & $93.93 \mathrm{ab}$ \\
\hline $\mathrm{V}_{4} \mathrm{~S}_{3}$ & $37.43 \mathrm{ab}$ & $51.32 \mathrm{ab}$ & $77.44 \mathrm{bc}$ & $90.61 \mathrm{a}-\mathrm{d}$ & $91.53 \mathrm{a}-\mathrm{e}$ & $91.95 \mathrm{a}-\mathrm{f}$ \\
\hline $\mathrm{V}_{5} \mathrm{~S}_{3}$ & $32.98 \mathrm{f}-\mathrm{h}$ & $42.68 \mathrm{~g}-\mathrm{i}$ & $70.58 \mathrm{f}-\mathrm{k}$ & 89.44 b-e & 90.18 b-g & 90.58 a-h \\
\hline $\mathrm{V}_{6} \mathrm{~S}_{3}$ & $37.44 \mathrm{ab}$ & $52.19 \mathrm{a}$ & $75.64 \mathrm{~b}-\mathrm{e}$ & $85.08 \mathrm{f}-\mathrm{i}$ & $86.44 \mathrm{f}-\mathrm{h}$ & $87.10 \mathrm{~g}-\mathrm{i}$ \\
\hline $\mathrm{V}_{1} \mathrm{~S}_{4}$ & 29.74 jk & 40.48 i-k & $73.01 \mathrm{c}-\mathrm{i}$ & 90.93 a-d & 91.96 a-e & $92.23 \mathrm{a}-\mathrm{e}$ \\
\hline $\mathrm{V}_{2} \mathrm{~S}_{4}$ & $33.06 \mathrm{e}-\mathrm{h}$ & $44.13 \mathrm{e}-\mathrm{i}$ & $80.20 \mathrm{a}$ & $91.71 \mathrm{a}-\mathrm{c}$ & $93.24 \mathrm{a}-\mathrm{c}$ & $93.81 \mathrm{a}-\mathrm{c}$ \\
\hline $\mathrm{V}_{3} \mathrm{~S}_{4}$ & 32.49 g-i & $41.48 \mathrm{~h}-\mathrm{j}$ & $73.26 \mathrm{c}-\mathrm{h}$ & $88.60 \mathrm{c}-\mathrm{f}$ & $89.56 \mathrm{~b}-\mathrm{g}$ & 90.03 b-h \\
\hline $\mathrm{V}_{4} \mathrm{~S}_{4}$ & $32.20 \mathrm{~g}-\mathrm{i}$ & $45.21 \mathrm{~d}-\mathrm{h}$ & $72.80 \mathrm{~d}-\mathrm{j}$ & $88.39 \mathrm{c}-\mathrm{f}$ & $89.00 \mathrm{c}-\mathrm{g}$ & $89.49 \mathrm{c}-\mathrm{h}$ \\
\hline $\mathrm{V}_{5} \mathrm{~S}_{4}$ & $27.94 \mathrm{k}$ & $36.25 \mathrm{k}$ & $67.45 \mathrm{k}$ & $87.66 \mathrm{~d}-\mathrm{g}$ & $88.51 \mathrm{~d}-\mathrm{h}$ & 89.08 d-h \\
\hline $\mathrm{V}_{6} \mathrm{~S}_{4}$ & $33.08 \mathrm{e}-\mathrm{h}$ & $45.55 \mathrm{c}-\mathrm{h}$ & $69.91 \mathrm{f}-\mathrm{k}$ & $80.08 \mathrm{j}$ & 80.95 i & $81.78 \mathrm{j}$ \\
\hline CV (\%) & 9.82 & 12.68 & 9.09 & 5.56 & 6.06 & 5.78 \\
\hline $\operatorname{LSD}(0.05)$ & 2.25 & 4.29 & 4.59 & 3.74 & 4.59 & 4.37 \\
\hline
\end{tabular}

Mean in a vertical column followed by same letter or without letter do not differ significantly at $5 \%$ level. 
However, in case of combined effect, seeds of BARI Gom-25 sown on November 15 $\left(\mathrm{V}_{3} \mathrm{~S}_{1}\right)$ resulted the highest number of tillers per plant at all ages of growth except at 30 and 60 days where, $\mathrm{V}_{5} \mathrm{~S}_{1}$ treatment produced maximum tillers per plant.

Table 2. Effects of sowing time on number of tillers per plant of six varieties of wheat at different ages.

\begin{tabular}{|c|c|c|c|c|c|c|}
\hline Treatments & 30 days & 45 days & 60 days & 75 days & 90 days & At harvest \\
\hline \multicolumn{7}{|l|}{ Variety (V) } \\
\hline $\mathrm{V}_{1}$ & $2.72 \mathrm{~b}$ & $6.56 \mathrm{ab}$ & $9.94 \mathrm{ab}$ & $10.59 \mathrm{ab}$ & $9.75 \mathrm{a}$ & $7.13 \mathrm{a}$ \\
\hline $\mathrm{V}_{2}$ & $2.16 \mathrm{c}$ & $5.77 \mathrm{~b}$ & $9.34 \mathrm{~b}$ & $9.88 \mathrm{ab}$ & $8.94 \mathrm{a}$ & $5.47 \mathrm{c}$ \\
\hline $\mathrm{V}_{3}$ & $2.31 \mathrm{bc}$ & $6.16 \mathrm{ab}$ & $8.75 \mathrm{~b}$ & $9.53 \mathrm{~b}$ & $9.00 \mathrm{a}$ & $6.38 \mathrm{~b}$ \\
\hline $\mathrm{V}_{4}$ & $2.66 \mathrm{~b}$ & $6.17 \mathrm{ab}$ & $9.84 \mathrm{ab}$ & $10.88 \mathrm{ab}$ & $9.69 \mathrm{a}$ & $6.25 \mathrm{~b}$ \\
\hline $\mathrm{V}_{5}$ & $3.31 \mathrm{a}$ & $6.97 \mathrm{a}$ & $11.09 \mathrm{a}$ & $11.41 \mathrm{a}$ & $9.84 \mathrm{a}$ & $6.31 \mathrm{~b}$ \\
\hline $\mathrm{V}_{6}$ & $2.56 \mathrm{bc}$ & $6.02 \mathrm{ab}$ & $9.69 \mathrm{~b}$ & $10.47 \mathrm{ab}$ & $9.66 \mathrm{a}$ & $6.75 \mathrm{ab}$ \\
\hline \multicolumn{7}{|c|}{ Sowing time $(\mathrm{S})$} \\
\hline $\mathrm{V}_{1}$ & $3.10 \mathrm{a}$ & $7.88 \mathrm{a}$ & $11.25 \mathrm{a}$ & $11.90 \mathrm{a}$ & $10.44 \mathrm{a}$ & $7.83 \mathrm{a}$ \\
\hline $\mathrm{V}_{2}$ & $2.19 \mathrm{~b}$ & $5.91 \mathrm{~b}$ & $9.31 \mathrm{~b}$ & $10.29 \mathrm{ab}$ & $9.13 \mathrm{a}$ & $6.96 \mathrm{~b}$ \\
\hline $\mathrm{V}_{3}$ & $3.10 \mathrm{a}$ & $6.73 \mathrm{~b}$ & $9.58 \mathrm{~b}$ & $10.13 \mathrm{~b}$ & $9.40 \mathrm{a}$ & $5.94 \mathrm{c}$ \\
\hline $\begin{array}{l}\mathrm{V}_{4} \\
(\mathrm{~V} \times \mathrm{S})\end{array}$ & $2.08 \mathrm{~b}$ & $4.58 \mathrm{c}$ & $8.96 \mathrm{~b}$ & $9.52 \mathrm{~b}$ & $8.96 \mathrm{a}$ & $4.79 \mathrm{~d}$ \\
\hline $\mathrm{V}_{1} \mathrm{~S}_{1}$ & $2.50 \mathrm{~g}-\mathrm{i}$ & $7.06 \mathrm{c}-\mathrm{e}$ & $10.13 \mathrm{~d}-\mathrm{f}$ & $11.13 \mathrm{ab}$ & $10.38 \mathrm{ab}$ & $8.25 \mathrm{a}$ \\
\hline $\mathrm{V}_{2} \mathrm{~S}_{1}$ & $2.38 \mathrm{~g}-\mathrm{i}$ & $5.94 \mathrm{f}-\mathrm{h}$ & $9.00 \mathrm{f}-\mathrm{i}$ & $9.75 \mathrm{~b}-\mathrm{g}$ & $9.75 \mathrm{~b}-\mathrm{d}$ & $6.88 \mathrm{c}-\mathrm{e}$ \\
\hline $\mathrm{V}_{3} \mathrm{~S}_{1}$ & $3.50 \mathrm{c}$ & $9.25 \mathrm{a}$ & $12.25 \mathrm{ab}$ & $12.50 \mathrm{a}$ & $12.13 \mathrm{a}$ & $8.38 \mathrm{a}$ \\
\hline $\mathrm{V}_{4} \mathrm{~S}_{1}$ & $3.25 \mathrm{~cd}$ & $8.38 \mathrm{ab}$ & $11.88 \mathrm{bc}$ & $11.38 \mathrm{ab}$ & $10.63 \mathrm{ab}$ & $7.75 a b$ \\
\hline $\mathrm{V}_{5} \mathrm{~S}_{1}$ & $4.00 \mathrm{~b}$ & $9.00 \mathrm{a}$ & $13.50 \mathrm{a}$ & $11.00 \mathrm{a}-\mathrm{c}$ & $9.88 \mathrm{bc}$ & $8.25 \mathrm{a}$ \\
\hline $\mathrm{V}_{6} \mathrm{~S}_{1}$ & $3.00 \mathrm{~d}-\mathrm{f}$ & $7.63 \mathrm{bc}$ & $10.75 c-e$ & $10.13 b-f$ & $9.88 \mathrm{bc}$ & $7.50 \mathrm{bc}$ \\
\hline $\mathrm{V}_{1} \mathrm{~S}_{2}$ & $2.75 \mathrm{e}-\mathrm{g}$ & $6.31 \mathrm{fg}$ & $10.25 \mathrm{~d}-\mathrm{f}$ & $10.13 \mathrm{~b}-\mathrm{f}$ & $9.25 \mathrm{~b}-\mathrm{f}$ & $7.88 \mathrm{ab}$ \\
\hline $\mathrm{V}_{2} \mathrm{~S}_{2}$ & $2.13 \mathrm{i}-\mathrm{k}$ & $7.06 \mathrm{c}-\mathrm{e}$ & $11.00 \mathrm{~b}-\mathrm{d}$ & 9.88 b-f & $9.13 \mathrm{~b}-\mathrm{f}$ & $6.25 \mathrm{ef}$ \\
\hline $\mathrm{V}_{3} \mathrm{~S}_{2}$ & 1.631 & $5.44 \mathrm{~g}-\mathrm{i}$ & $8.25 \mathrm{~h}-\mathrm{j}$ & $8.63 \mathrm{f}-\mathrm{j}$ & $8.88 \mathrm{~b}-\mathrm{f}$ & $7.38 \mathrm{bc}$ \\
\hline $\mathrm{V}_{4} \mathrm{~S}_{2}$ & $2.13 \mathrm{i}-\mathrm{k}$ & $5.69 \mathrm{f}-\mathrm{h}$ & $7.88 \mathrm{i}-\mathrm{k}$ & $8.88 \mathrm{f}-\mathrm{i}$ & $8.13 \mathrm{c}-\mathrm{f}$ & $6.38 \mathrm{~d}-\mathrm{f}$ \\
\hline $\mathrm{V}_{5} \mathrm{~S}_{2}$ & $2.13 \mathrm{i}-\mathrm{k}$ & $5.06 \mathrm{~h}-\mathrm{j}$ & $7.88 \mathrm{i}-\mathrm{k}$ & $9.25 \mathrm{~d}-\mathrm{h}$ & $9.13 \mathrm{~b}-\mathrm{f}$ & $6.88 \mathrm{c}-\mathrm{e}$ \\
\hline $\mathrm{V}_{6} \mathrm{~S}_{2}$ & $2.38 \mathrm{~g}-\mathrm{i}$ & $5.88 \mathrm{f}-\mathrm{h}$ & 9.50 e-h & $10.75 \mathrm{~b}-\mathrm{d}$ & $10.25 \mathrm{ab}$ & $7.00 \mathrm{~cd}$ \\
\hline $\mathrm{V}_{1} \mathrm{~S}_{3}$ & $3.13 \mathrm{c}-\mathrm{d}$ & $7.44 \mathrm{~b}-\mathrm{d}$ & $10.38 \mathrm{de}$ & $10.00 \mathrm{~b}-\mathrm{f}$ & $10.13 \mathrm{a}-\mathrm{c}$ & $6.88 \mathrm{c}-\mathrm{e}$ \\
\hline $\mathrm{V}_{2} \mathrm{~S}_{3}$ & $2.25 \mathrm{~h}-\mathrm{j}$ & $6.00 \mathrm{f}-\mathrm{h}$ & $7.75 \mathrm{i}-\mathrm{k}$ & $7.63 \mathrm{~h}-\mathrm{j}$ & 7.63 ef & $4.38 \mathrm{jk}$ \\
\hline $\mathrm{V}_{3} \mathrm{~S}_{3}$ & $2.38 \mathrm{~g}-\mathrm{i}$ & $5.56 \mathrm{f}-\mathrm{h}$ & $6.88 \mathrm{k}$ & $7.63 \mathrm{~h}-\mathrm{j}$ & $7.25 \mathrm{f}$ & $5.00 \mathrm{~h}-\mathrm{j}$ \\
\hline $\mathrm{V}_{4} \mathrm{~S}_{3}$ & $3.13 \mathrm{c}-\mathrm{e}$ & $6.38 \mathrm{~d}-\mathrm{g}$ & $11.00 \mathrm{bd}$ & $9.00 \mathrm{e}-\mathrm{i}$ & $10.38 \mathrm{ab}$ & $5.50 \mathrm{gh}$ \\
\hline $\mathrm{V}_{5} \mathrm{~S}_{3}$ & $4.50 \mathrm{a}$ & $8.38 \mathrm{ab}$ & $11.13 \mathrm{~b}-\mathrm{d}$ & $9.38 \mathrm{c}-\mathrm{g}$ & $10.63 \mathrm{ab}$ & $6.00 \mathrm{fg}$ \\
\hline $\mathrm{V}_{6} \mathrm{~S}_{3}$ & $3.25 \mathrm{~cd}$ & $6.63 c-f$ & $10.38 \mathrm{de}$ & 10.63 & $10.38 \mathrm{ab}$ & $7.88 \mathrm{ab}$ \\
\hline $\mathrm{V}_{1} \mathrm{~S}_{4}$ & $2.50 \mathrm{~g}-\mathrm{i}$ & $5.44 \mathrm{~g}-\mathrm{i}$ & $9.00 \mathrm{f}-\mathrm{i}$ & $8.88 \mathrm{f}-\mathrm{i}$ & $9.25 \mathrm{~b}-\mathrm{f}$ & $5.50 \mathrm{gh}$ \\
\hline $\mathrm{V}_{2} \mathrm{~S}_{4}$ & $1.88 \mathrm{j}-1$ & $4.06 \mathrm{j}$ & $9.63 \mathrm{e}-\mathrm{g}$ & $7.38 \mathrm{ij}$ & $9.25 \mathrm{~b}-\mathrm{f}$ & $4.38 \mathrm{jk}$ \\
\hline $\mathrm{V}_{3} \mathrm{~S}_{4}$ & $1.75 \mathrm{kl}$ & $4.38 \mathrm{ij}$ & $7.63 \mathrm{jk}$ & $7.13 \mathrm{j}$ & $7.75 \mathrm{~d}-\mathrm{f}$ & $4.75 \mathrm{i}-\mathrm{k}$ \\
\hline $\mathrm{V}_{4} \mathrm{~S}_{4}$ & $2.13 \mathrm{i}-\mathrm{k}$ & $4.25 \mathrm{j}$ & $8.63 \mathrm{~g}-\mathrm{j}$ & $9.38 \mathrm{c}-\mathrm{g}$ & $9.63 \mathrm{~b}-\mathrm{e}$ & $5.38 \mathrm{~g}-\mathrm{i}$ \\
\hline $\mathrm{V}_{5} \mathrm{~S}_{4}$ & $2.63 \mathrm{f}-\mathrm{h}$ & $5.44 \mathrm{~g}-\mathrm{i}$ & $10.25 \mathrm{~d}-\mathrm{f}$ & $7.38 \mathrm{ij}$ & $9.75 b-d$ & $4.13 \mathrm{k}$ \\
\hline $\mathrm{V}_{6} \mathrm{~S}_{4}$ & 1.631 & $3.94 \mathrm{j}$ & $8.63 \mathrm{~g}-\mathrm{j}$ & $8.13 \mathrm{~g}-\mathrm{j}$ & $8.13 \mathrm{c}-\mathrm{f}$ & $4.63 \mathrm{jk}$ \\
\hline CV $(\%)$ & 13.88 & 9.44 & 10.76 & 10.31 & 13.99 & 13.87 \\
\hline $\operatorname{LSD}(0.05)$ & 0.43 & 1.14 & 1.35 & 1.67 & 2.08 & 0.71 \\
\hline
\end{tabular}

Mean in a vertical column followed by same letter or without letter do not differ significantly at $5 \%$ level.

Results obtained from the present study corroborate with the findings of Jahan and Adam (2015) on BARI Gom-25. Ansary et al. (1989) also reported that delay sowing caused reduction in the number of tillers in wheat. However, Baloch et al. (2010) obtained non-significant effect on the number of tillers per unit area with sowing time. 
Table 3. Effects of sowing time on number of leaves per plant of six varieties of wheat at different ages.

\begin{tabular}{|c|c|c|c|c|c|c|}
\hline Treatments & 30 days & 45 days & 60 days & 75 days & 90 days & At harvest \\
\hline \multicolumn{7}{|l|}{ Variety (V) } \\
\hline $\mathrm{V}_{1}$ & $12.25 \mathrm{ab}$ & $24.36 \mathrm{a}$ & $55.04 \mathrm{a}$ & $42.25 \mathrm{a}$ & $28.47 \mathrm{a}$ & $17.78 \mathrm{a}$ \\
\hline $\mathrm{V}_{2}$ & $11.16 \mathrm{c}$ & $22.48 \mathrm{a}$ & $44.97 \mathrm{c}$ & $40.66 \mathrm{a}$ & $26.03 \mathrm{a}$ & $13.59 \mathrm{c}$ \\
\hline $\mathrm{V}_{3}$ & $11.81 \mathrm{a}-\mathrm{c}$ & $22.81 \mathrm{a}$ & $45.38 \mathrm{c}$ & $40.19 \mathrm{a}$ & $27.16 \mathrm{a}$ & $15.59 \mathrm{bc}$ \\
\hline $\mathrm{V}_{4}$ & $12.50 \mathrm{ab}$ & $22.88 \mathrm{a}$ & $47.50 \mathrm{bc}$ & $43.03 \mathrm{a}$ & $27.16 \mathrm{a}$ & $15.53 \mathrm{bc}$ \\
\hline $\mathrm{V}_{5}$ & $12.81 \mathrm{a}$ & $24.59 \mathrm{a}$ & $51.16 \mathrm{ab}$ & $43.72 \mathrm{a}$ & $26.16 \mathrm{a}$ & $15.38 \mathrm{bc}$ \\
\hline $\mathrm{V}_{6}$ & $11.72 \mathrm{bc}$ & $22.05 \mathrm{a}$ & $44.31 \mathrm{c}$ & $39.50 \mathrm{a}$ & $27.78 \mathrm{a}$ & $16.81 \mathrm{ab}$ \\
\hline \multicolumn{7}{|c|}{ Sowing time (S) } \\
\hline $\mathrm{V}_{1}$ & $13.81 \mathrm{a}$ & $27.97 \mathrm{a}$ & $55.04 \mathrm{a}$ & $50.56 \mathrm{a}$ & $33.60 \mathrm{a}$ & $20.06 \mathrm{a}$ \\
\hline $\mathrm{V}_{2}$ & $11.04 \mathrm{~b}$ & $20.60 \mathrm{~b}$ & $45.75 \mathrm{~b}$ & $40.19 \mathrm{~b}$ & $27.25 \mathrm{~b}$ & $16.38 \mathrm{~b}$ \\
\hline $\mathrm{V}_{3}$ & $13.25 \mathrm{a}$ & $26.44 \mathrm{a}$ & $47.40 \mathrm{~b}$ & $39.71 \mathrm{~b}$ & $25.69 \mathrm{~b}$ & $14.27 \mathrm{c}$ \\
\hline $\begin{array}{l}\mathrm{V}_{4} \\
(\mathrm{~V} \times \mathrm{S})\end{array}$ & $10.06 \mathrm{~b}$ & $17.77 \mathrm{~b}$ & $39.08 \mathrm{c}$ & $35.77 \mathrm{~b}$ & $21.96 \mathrm{~b}$ & $12.42 \mathrm{c}$ \\
\hline $\mathrm{V}_{1} \mathrm{~S}_{1}$ & $11.50 \mathrm{e}-\mathrm{g}$ & $26.19 \mathrm{~b}-\mathrm{d}$ & $51.00 \mathrm{~d}-\mathrm{f}$ & $43.75 \mathrm{a}-\mathrm{c}$ & $35.75 \mathrm{ab}$ & $22.63 \mathrm{a}$ \\
\hline $\mathrm{V}_{2} \mathrm{~S}_{1}$ & $10.63 \mathrm{gh}$ & $23.31 \mathrm{~d}-\mathrm{f}$ & $47.00 \mathrm{f}-\mathrm{h}$ & $40.13 \mathrm{~b}-\mathrm{d}$ & $30.88 \mathrm{~b}-\mathrm{d}$ & $16.75 \mathrm{e}-\mathrm{g}$ \\
\hline $\mathrm{V}_{3} \mathrm{~S}_{1}$ & $16.13 \mathrm{a}$ & $31.44 \mathrm{a}$ & $60.00 \mathrm{~b}$ & $48.13 \mathrm{a}$ & $38.50 \mathrm{a}$ & $23.13 \mathrm{a}$ \\
\hline $\mathrm{V}_{4} \mathrm{~S}_{1}$ & $14.88 \mathrm{~b}$ & $29.31 \mathrm{a}-\mathrm{c}$ & $57.00 \mathrm{bc}$ & $46.69 \mathrm{ab}$ & $34.13 \mathrm{a}-\mathrm{c}$ & $19.25 \mathrm{~b}-\mathrm{d}$ \\
\hline $\mathrm{V}_{5} \mathrm{~S}_{1}$ & $16.25 \mathrm{a}$ & $31.00 \mathrm{a}$ & $64.63 \mathrm{a}$ & $46.50 \mathrm{ab}$ & $31.75 \mathrm{~b}-\mathrm{d}$ & $20.38 \mathrm{~b}$ \\
\hline $\mathrm{V}_{6} \mathrm{~S}_{1}$ & $13.50 \mathrm{c}$ & $26.56 b-d$ & $50.63 \mathrm{~d}-\mathrm{f}$ & $39.75 \mathrm{~b}-\mathrm{d}$ & $30.63 \mathrm{~b}-\mathrm{e}$ & $18.25 \mathrm{c}-\mathrm{e}$ \\
\hline $\mathrm{V}_{1} \mathrm{~S}_{2}$ & $13.38 \mathrm{~cd}$ & $23.50 \mathrm{de}$ & 49.75 ef & $35.50 \mathrm{~d}-\mathrm{h}$ & $28.00 \mathrm{~d}-\mathrm{g}$ & $18.50 \mathrm{~b}-\mathrm{e}$ \\
\hline $\mathrm{V}_{2} \mathrm{~S}_{2}$ & $13.00 \mathrm{~cd}$ & $24.06 \mathrm{~d}$ & $55.00 \mathrm{~cd}$ & $39.75 \mathrm{~b}-\mathrm{d}$ & $29.38 c-f$ & $15.25 \mathrm{gh}$ \\
\hline $\mathrm{V}_{3} \mathrm{~S}_{2}$ & $10.38 \mathrm{hi}$ & $19.44 \mathrm{f}-\mathrm{h}$ & $43.00 \mathrm{~h}-\mathrm{j}$ & $32.38 \mathrm{~b}-\mathrm{d}$ & $26.13 \mathrm{~d}-\mathrm{i}$ & $14.88 \mathrm{gh}$ \\
\hline $\mathrm{V}_{4} \mathrm{~S}_{2}$ & $10.75 \mathrm{ef}$ & $19.38 \mathrm{gh}$ & $41.38 \mathrm{i}-\mathrm{k}$ & $34.38 \mathrm{~d}-\mathrm{h}$ & $24.63 e-j$ & $15.50 \mathrm{gh}$ \\
\hline $\mathrm{V}_{5} \mathrm{~S}_{2}$ & 7.88 & $17.38 \mathrm{gh}$ & $43.50 \mathrm{hi}$ & $36.13 \mathrm{~d}-\mathrm{g}$ & $26.88 \mathrm{~d}-\mathrm{h}$ & $16.75 \mathrm{e}-\mathrm{g}$ \\
\hline $\mathrm{V}_{6} \mathrm{~S}_{2}$ & $10.88 \mathrm{f}-\mathrm{h}$ & $19.88 \mathrm{e}-\mathrm{g}$ & $41.88 \mathrm{i}-\mathrm{k}$ & $36.63 \mathrm{c}-\mathrm{f}$ & $28.50 \mathrm{c}-\mathrm{g}$ & $17.38 \mathrm{~d}-\mathrm{f}$ \\
\hline $\mathrm{V}_{1} \mathrm{~S}_{3}$ & $12.38 \mathrm{de}$ & $28.88 \mathrm{a}-\mathrm{c}$ & $49.75 \mathrm{ef}$ & $36.88 \mathrm{c}-\mathrm{e}$ & $26.88 \mathrm{~d}-\mathrm{h}$ & $16.25 \mathrm{fg}$ \\
\hline $\mathrm{V}_{2} \mathrm{~S}_{3}$ & $11.75 \mathrm{ef}$ & $24.94 \mathrm{~d}$ & $41.50 \mathrm{i}-\mathrm{k}$ & 32.50 e-h & $23.75 \mathrm{f}-\mathrm{j}$ & $11.13 \mathrm{j}$ \\
\hline $\mathrm{V}_{3} \mathrm{~S}_{3}$ & $11.38 \mathrm{e}-\mathrm{h}$ & $23.44 \mathrm{de}$ & $40.75 \mathrm{i}-1$ & $29.50 \mathrm{f}-\mathrm{h}$ & $22.50 \mathrm{~g}-\mathrm{j}$ & $10.75 \mathrm{j}$ \\
\hline $\mathrm{V}_{4} \mathrm{~S}_{3}$ & $14.00 \mathrm{bc}$ & $25.50 \mathrm{~cd}$ & $52.25 \mathrm{de}$ & $36.50 \mathrm{~d}-\mathrm{g}$ & $24.00 \mathrm{f}-\mathrm{j}$ & $13.50 \mathrm{hi}$ \\
\hline $\mathrm{V}_{5} \mathrm{~S}_{3}$ & $16.38 \mathrm{a}$ & $29.94 \mathrm{ab}$ & $51.50 \mathrm{~d}-\mathrm{f}$ & $35.25 \mathrm{~d}-\mathrm{g}$ & $26.00 \mathrm{~d}-\mathrm{j}$ & $14.13 \mathrm{~h}$ \\
\hline $\mathrm{V}_{6} \mathrm{~S}_{3}$ & $13.63 \mathrm{c}$ & $25.94 \mathrm{~cd}$ & $48.63 \mathrm{e}-\mathrm{g}$ & $40.00 \mathrm{~b}-\mathrm{d}$ & $31.00 \mathrm{~b}-\mathrm{d}$ & $19.88 \mathrm{bc}$ \\
\hline $\mathrm{V}_{1} \mathrm{~S}_{4}$ & $11.75 \mathrm{ef}$ & $18.88 \mathrm{gh}$ & $39.88 \mathrm{j}-\mathrm{m}$ & $34.25 \mathrm{~d}-\mathrm{g}$ & $23.25 \mathrm{~g}-\mathrm{j}$ & $13.75 \mathrm{hi}$ \\
\hline $\mathrm{V}_{2} \mathrm{~S}_{4}$ & $9.25 \mathrm{j}$ & $17.63 \mathrm{gh}$ & $36.38 \mathrm{~lm}$ & 30.50 e-h & $23.25 \mathrm{~g}-\mathrm{j}$ & $11.25 \mathrm{j}$ \\
\hline $\mathrm{V}_{3} \mathrm{~S}_{4}$ & $9.38 \mathrm{ij}$ & $16.94 \mathrm{gh}$ & $37.75 \mathrm{k}-\mathrm{m}$ & $28.75 \mathrm{~h}$ & $20.13 \mathrm{ij}$ & $13.63 \mathrm{hi}$ \\
\hline $\mathrm{V}_{4} \mathrm{~S}_{4}$ & $10.38 \mathrm{hi}$ & $17.31 \mathrm{gh}$ & $39.38 \mathrm{j}-\mathrm{m}$ & $33.44 \mathrm{~d}-\mathrm{g}$ & $21.50 \mathrm{~h}-\mathrm{j}$ & $13.88 \mathrm{~h}$ \\
\hline $\mathrm{V}_{5} \mathrm{~S}_{4}$ & $10.75 \mathrm{f}-\mathrm{h}$ & $20.06 \mathrm{e}-\mathrm{g}$ & $45.00 \mathrm{~g}-\mathrm{i}$ & 30.38 e-h & $25.88 \mathrm{~d}-\mathrm{j}$ & $10.25 \mathrm{j}$ \\
\hline $\mathrm{V}_{6} \mathrm{~S}_{4}$ & $8.88 \mathrm{jk}$ & $15.81 \mathrm{~h}$ & $36.13 \mathrm{~m}$ & $29.38 \mathrm{gh}$ & $20.00 \mathrm{j}$ & $11.75 \mathrm{ij}$ \\
\hline CV (\%) & 10.81 & 15.87 & 16.01 & 14.02 & 7.92 & 15.72 \\
\hline $\operatorname{LSD}(0.05)$ & 1.00 & 3.88 & 4.57 & 7.15 & 6.10 & 2.02 \\
\hline
\end{tabular}

Mean in a vertical column followed by same letter or without letter do not differ significantly at $5 \%$ level.

Results presented in Table 3 revealed that number of leaves per plant had similar trend as obtained in number of tillers per plant but with few exceptions. The maximum number of leaves per plant was recorded from November 15 sown wheat $\left(S_{1}\right)$ throughout the growth stage although, statistically at par with November 29 sown wheat $\left(\mathrm{S}_{3}\right)$ at 30 and 45 days. The combined effect of $\mathrm{V}_{3} \mathrm{~S}_{1}$ treatment also resulted the highest number of leaves per plant throughout life span except at 30 and 60 days. Results obtained from this investigation were found to be consistent with the findings of Jahan and Adam (2015) where, they recorded maximum number of leaves per plant from November 14 sown BARI Gom-25 in most of the growth ages. 
Table 4 showed that BARI Gom-25 $\left(\mathrm{V}_{3}\right)$ produced the highest total dry matter (TDM) throughout the growth stages but with exception of 30 and 60 days whereas, in case of sowing time the maximum value was obtained from November 15 sown wheat $\left(S_{1}\right)$ at all ages of growth. Similarly, maximum TDM was noted from November 15 sown BARI Gom-25 $\left(\mathrm{V}_{3} \mathrm{~S}_{1}\right)$ at all growth stages and found significantly different from rest of the combined treatments at 60,75 and 90 days. The production of higher TDM per plant of BARI Gom-25 might be due to increased vegetative growth on optimum time of sowing.

Table 4. Effects of sowing time on total dry matter per plant of six varieties of wheat at different ages.

\begin{tabular}{|c|c|c|c|c|c|c|}
\hline Treatments & 30 days & 45 days & 60 days & 75 days & 90 days & At harvest \\
\hline \multicolumn{7}{|l|}{ Variety (V) } \\
\hline $\mathrm{V}_{1}$ & $0.66 \mathrm{ab}$ & $1.87 \mathrm{~b}$ & $3.86 \mathrm{a}$ & $16.56 \mathrm{~b}$ & $19.54 \mathrm{~b}$ & $29.42 \mathrm{a}$ \\
\hline $\mathrm{V}_{2}$ & $0.70 \mathrm{ab}$ & $2.16 \mathrm{a}$ & $4.09 \mathrm{a}$ & $18.50 \mathrm{a}$ & $21.55 \mathrm{a}$ & $31.50 \mathrm{a}$ \\
\hline $\mathrm{V}_{3}$ & $0.71 \mathrm{ab}$ & $2.21 \mathrm{a}$ & $4.12 \mathrm{a}$ & $18.62 \mathrm{a}$ & $21.90 \mathrm{a}$ & $31.82 \mathrm{a}$ \\
\hline $\mathrm{V}_{4}$ & $0.76 \mathrm{a}$ & $1.96 \mathrm{ab}$ & $4.30 \mathrm{a}$ & $17.67 \mathrm{ab}$ & $20.18 b$ & $29.96 \mathrm{a}$ \\
\hline $\mathrm{V}_{5}$ & $0.63 \mathrm{~b}$ & $2.07 \mathrm{ab}$ & $4.35 \mathrm{a}$ & $15.14 \mathrm{c}$ & $18.26 \mathrm{c}$ & $28.04 \mathrm{a}$ \\
\hline $\mathrm{V}_{6}$ & $0.64 \mathrm{~b}$ & $2.05 \mathrm{ab}$ & $3.99 \mathrm{a}$ & $15.10 \mathrm{c}$ & $17.99 \mathrm{c}$ & $28.05 \mathrm{a}$ \\
\hline \multicolumn{7}{|c|}{ Sowing time (S) } \\
\hline $\mathrm{V}_{1}$ & $0.77 \mathrm{a}$ & $2.61 \mathrm{a}$ & $4.65 \mathrm{a}$ & $18.43 \mathrm{a}$ & $21.12 \mathrm{a}$ & $34.29 \mathrm{a}$ \\
\hline $\mathrm{V}_{2}$ & $0.66 \mathrm{bc}$ & $1.48 \mathrm{~b}$ & $3.07 \mathrm{~b}$ & $16.50 \mathrm{bc}$ & $20.55 \mathrm{a}$ & $29.01 \mathrm{~b}$ \\
\hline $\mathrm{V}_{3}$ & $0.74 \mathrm{ab}$ & $2.55 \mathrm{a}$ & $4.43 \mathrm{a}$ & $17.37 \mathrm{ab}$ & $20.14 \mathrm{a}$ & $28.85 \mathrm{~b}$ \\
\hline $\begin{array}{l}V_{4} \\
(\mathrm{~V} \times \mathrm{S})\end{array}$ & $0.57 \mathrm{c}$ & $1.57 \mathrm{~b}$ & $4.33 \mathrm{a}$ & $15.42 \mathrm{c}$ & $17.79 \mathrm{~b}$ & $27.04 \mathrm{~b}$ \\
\hline $\mathrm{V}_{1} \mathrm{~S}_{1}$ & $0.76 \mathrm{a}-\mathrm{c}$ & $2.06 \mathrm{e}$ & $3.96 \mathrm{de}$ & $15.79 \mathrm{fg}$ & $18.73 \mathrm{k}-\mathrm{n}$ & $33.03 \mathrm{~b}-\mathrm{d}$ \\
\hline $\mathrm{V}_{2} \mathrm{~S}_{1}$ & $0.78 \mathrm{ab}$ & $2.84 \mathrm{ab}$ & $4.78 b c$ & $19.83 \mathrm{~b}$ & $23.43 \mathrm{~b}$ & $35.83 \mathrm{ab}$ \\
\hline $\mathrm{V}_{3} \mathrm{~S}_{1}$ & $0.81 \mathrm{a}$ & $3.01 \mathrm{a}$ & $5.70 \mathrm{a}$ & $23.07 \mathrm{a}$ & $25.66 \mathrm{a}$ & $39.01 \mathrm{a}$ \\
\hline $\mathrm{V}_{4} \mathrm{~S}_{1}$ & $0.80 \mathrm{a}$ & $2.36 \mathrm{~d}$ & $4.21 \mathrm{c}-\mathrm{e}$ & $18.67 \mathrm{bc}$ & 19.97 e-j & $34.20 \mathrm{bc}$ \\
\hline $\mathrm{V}_{5} \mathrm{~S}_{1}$ & $0.69 \mathrm{~b}-\mathrm{d}$ & $2.84 \mathrm{ab}$ & $4.84 \mathrm{~b}$ & $18.47 \mathrm{c}$ & $20.08 \mathrm{e}-\mathrm{i}$ & $32.97 \mathrm{~b}-\mathrm{e}$ \\
\hline $\mathrm{V}_{6} \mathrm{~S}_{1}$ & $0.78 \mathrm{ab}$ & $2.54 \mathrm{~cd}$ & $4.40 \mathrm{~b}-\mathrm{e}$ & $14.77 \mathrm{gh}$ & $18.86 \mathrm{j}-\mathrm{n}$ & $30.70 \mathrm{c}-\mathrm{g}$ \\
\hline $\mathrm{V}_{1} \mathrm{~S}_{2}$ & $0.67 \mathrm{~cd}$ & $1.11 \mathrm{~h}$ & $2.57 \mathrm{f}$ & $14.13 \mathrm{hi}$ & $19.18 \mathrm{i}-\mathrm{n}$ & $29.12 \mathrm{e}-\mathrm{i}$ \\
\hline $\mathrm{V}_{2} \mathrm{~S}_{2}$ & $0.77 \mathrm{a}-\mathrm{c}$ & $1.63 \mathrm{fg}$ & $2.86 \mathrm{f}$ & $17.69 \mathrm{c}-\mathrm{e}$ & 21.04 e-e & 32.17 b-f \\
\hline $\mathrm{V}_{3} \mathrm{~S}_{2}$ & $0.79 \mathrm{ab}$ & $1.76 \mathrm{f}$ & $2.28 \mathrm{f}$ & $15.62 \mathrm{fg}$ & $21.82 \mathrm{c}$ & $30.75 \mathrm{c}-\mathrm{g}$ \\
\hline $\mathrm{V}_{4} \mathrm{~S}_{2}$ & $0.78 \mathrm{ab}$ & $1.57 \mathrm{f}-\mathrm{h}$ & $4.20 \mathrm{de}$ & $17.75 \mathrm{c}-\mathrm{e}$ & $21.07 \mathrm{c}-\mathrm{e}$ & $29.35 \mathrm{~d}-\mathrm{i}$ \\
\hline $\mathrm{V}_{5} \mathrm{~S}_{2}$ & $0.54 \mathrm{fg}$ & $1.35 \mathrm{~h}$ & $3.83 \mathrm{e}$ & $17.09 \mathrm{de}$ & $20.41 \mathrm{~d}-\mathrm{h}$ & $26.68 \mathrm{~h}-\mathrm{j}$ \\
\hline $\mathrm{V}_{6} \mathrm{~S}_{2}$ & $0.41 \mathrm{~h}$ & $1.46 \mathrm{gh}$ & $2.69 \mathrm{f}$ & $16.72 \mathrm{ef}$ & $19.80 \mathrm{f}-\mathrm{k}$ & $25.98 \mathrm{ij}$ \\
\hline $\mathrm{V}_{1} \mathrm{~S}_{3}$ & $0.65 \mathrm{de}$ & $2.52 \mathrm{~cd}$ & $4.47 \mathrm{~b}-\mathrm{d}$ & $18.35 \mathrm{~cd}$ & 20.94 c-f & $27.69 \mathrm{~g}-\mathrm{i}$ \\
\hline $\mathrm{V}_{2} \mathrm{~S}_{3}$ & $0.77 \mathrm{a}-\mathrm{c}$ & $2.44 \mathrm{~cd}$ & $4.46 \mathrm{~b}-\mathrm{d}$ & $18.08 \mathrm{~cd}$ & $21.34 \mathrm{~cd}$ & $29.91 \mathrm{~d}-\mathrm{h}$ \\
\hline $\mathrm{V}_{3} \mathrm{~S}_{3}$ & $0.78 \mathrm{ab}$ & $2.59 \mathrm{~b}-\mathrm{d}$ & $4.40 \mathrm{~b}-\mathrm{e}$ & $18.26 \mathrm{~cd}$ & $20.60 \mathrm{~d}-\mathrm{g}$ & $29.15 \mathrm{e}-\mathrm{i}$ \\
\hline $\mathrm{V}_{4} \mathrm{~S}_{3}$ & $0.80 \mathrm{a}$ & $2.52 \mathrm{~cd}$ & $4.41 \mathrm{~b}-\mathrm{e}$ & $18.52 \mathrm{c}$ & $21.49 \mathrm{~cd}$ & $28.29 \mathrm{~g}-\mathrm{i}$ \\
\hline $\mathrm{V}_{5} \mathrm{~S}_{3}$ & $0.72 \mathrm{a}-\mathrm{d}$ & $2.64 \mathrm{bc}$ & $4.37 \mathrm{~b}-\mathrm{e}$ & $12.93 \mathrm{ij}$ & $18.14 \mathrm{n}$ & $25.78 \mathrm{ij}$ \\
\hline $\mathrm{V}_{6} \mathrm{~S}_{3}$ & $0.74 \mathrm{a}-\mathrm{d}$ & $2.59 \mathrm{~b}-\mathrm{d}$ & $4.46 \mathrm{~b}-\mathrm{d}$ & $18.10 \mathrm{~cd}$ & $18.36 \ln$ & $32.25 \mathrm{~b}-\mathrm{e}$ \\
\hline $\mathrm{V}_{1} \mathrm{~S}_{4}$ & $0.55 \mathrm{e}-\mathrm{g}$ & $1.77 \mathrm{f}$ & $4.44 \mathrm{~b}-\mathrm{d}$ & $17.97 \mathrm{c}-\mathrm{e}$ & $19.32 \mathrm{~h}-\mathrm{m}$ & 27.83 g-i \\
\hline $\mathrm{V}_{2} \mathrm{~S}_{4}$ & $0.49 \mathrm{gh}$ & $1.75 \mathrm{f}$ & $4.27 \mathrm{~b}-\mathrm{e}$ & $18.40 \mathrm{c}$ & 20.40 d-h & $28.07 \mathrm{~g}-\mathrm{i}$ \\
\hline $\mathrm{V}_{3} \mathrm{~S}_{4}$ & $0.48 \mathrm{gh}$ & $1.46 \mathrm{gh}$ & $4.11 \mathrm{de}$ & $17.54 \mathrm{c}-\mathrm{e}$ & $19.50 \mathrm{~g}-1$ & $28.35 \mathrm{f}-\mathrm{i}$ \\
\hline $\mathrm{V}_{4} \mathrm{~S}_{4}$ & $0.67 \mathrm{~cd}$ & $1.38 \mathrm{gh}$ & $4.37 \mathrm{~b}-\mathrm{e}$ & $15.74 \mathrm{fg}$ & $18.18 \mathrm{mn}$ & $28.01 \mathrm{~g}-\mathrm{i}$ \\
\hline $\mathrm{V}_{5} \mathrm{~S}_{4}$ & $0.56 \mathrm{e}-\mathrm{g}$ & $1.45 \mathrm{gh}$ & $4.36 \mathrm{~b}-\mathrm{e}$ & $12.07 \mathrm{jk}$ & 14.39 o & $26.74 \mathrm{~h}-\mathrm{j}$ \\
\hline $\mathrm{V}_{6} \mathrm{~S}_{4}$ & $0.64 \mathrm{~d}-\mathrm{f}$ & $1.63 \mathrm{fg}$ & $4.41 \mathrm{~b}-\mathrm{e}$ & $10.81 \mathrm{k}$ & $14.95 \mathrm{o}$ & $23.27 \mathrm{j}$ \\
\hline CV $(\%)$ & 11.24 & 9.61 & 11.89 & 16.43 & 12.44 & 6.89 \\
\hline $\operatorname{LSD}(0.05)$ & 0.10 & 0.25 & 0.59 & 1.27 & 1.16 & 3.86 \\
\hline
\end{tabular}

Mean in a vertical column followed by same letter or without letter do not differ significantly at $5 \%$ level. 
Data obtained on yield attributes and yield are presented in Table 5. In case of varietal effect, the maximum number of grains per plant, dry weight of spike, 1000-grain weight, yield per plant, yield per hectare and harvest index were recorded from BARI Gom-25 $\left(\mathrm{V}_{3}\right)$.

Table 5. Effects of sowing time on yield attributes and yield of six varieties of wheat.

\begin{tabular}{|c|c|c|c|c|c|c|c|c|c|c|}
\hline $\begin{array}{l}\text { Treat- } \\
\text { ments }\end{array}$ & $\begin{array}{c}\text { No. of } \\
\text { effective } \\
\text { tillers/plant }\end{array}$ & $\begin{array}{l}\text { No. of non- } \\
\text { effective } \\
\text { tillers/plant }\end{array}$ & $\begin{array}{l}\text { No. of } \\
\text { grains/ } \\
\text { spike }\end{array}$ & $\begin{array}{c}\text { No. of } \\
\text { grains/ plant }\end{array}$ & $\begin{array}{l}\text { Length } \\
\text { of spike } \\
(\mathrm{cm})\end{array}$ & $\begin{array}{l}\text { Dry weight } \\
\text { of spikes } \\
(\mathrm{g})\end{array}$ & $\begin{array}{c}1000- \\
\text { grain } \\
\text { weight } \\
(\mathrm{g})\end{array}$ & $\begin{array}{l}\text { Yield/ } \\
\text { plant } \\
(\mathrm{g})\end{array}$ & $\begin{array}{l}\text { Yield/ } \\
\text { ha } \\
\text { (t) }\end{array}$ & $\begin{array}{l}\mathrm{HI} \\
(\%)\end{array}$ \\
\hline \multicolumn{11}{|c|}{ Variety (V) } \\
\hline $\mathrm{V}_{1}$ & $6.59 \mathrm{a}$ & $0.53 \mathrm{a}$ & $24.06 \mathrm{c}$ & $158.73 \mathrm{a}$ & $8.81 \mathrm{c}$ & $17.10 \mathrm{~cd}$ & $45.08 \mathrm{~b}$ & $7.44 \mathrm{c}$ & $3.72 \mathrm{c}$ & $25.39 \mathrm{ab}$ \\
\hline $\mathrm{V}_{2}$ & $5.25 \mathrm{c}$ & $0.22 \mathrm{a}$ & $32.08 \mathrm{a}$ & $165.62 \mathrm{a}$ & $10.37 \mathrm{a}$ & $19.12 \mathrm{ab}$ & $48.91 \mathrm{a}$ & $8.38 \mathrm{ab}$ & $4.19 \mathrm{ab}$ & $26.48 \mathrm{a}$ \\
\hline $\mathrm{V}_{3}$ & $6.19 \mathrm{ab}$ & $0.19 \mathrm{a}$ & $28.28 \mathrm{~b}$ & $172.79 \mathrm{a}$ & $9.49 \mathrm{~b}$ & $20.70 \mathrm{a}$ & $49.04 \mathrm{a}$ & $8.75 \mathrm{a}$ & $4.38 \mathrm{a}$ & $27.48 \mathrm{a}$ \\
\hline $\mathrm{V}_{4}$ & $5.88 \mathrm{~b}$ & $0.38 \mathrm{a}$ & $28.53 \mathrm{~b}$ & $168.12 \mathrm{a}$ & $8.95 \mathrm{c}$ & $18.41 \mathrm{bc}$ & $42.18 \mathrm{~d}$ & $7.39 \mathrm{c}$ & $3.69 \mathrm{c}$ & $24.76 \mathrm{ab}$ \\
\hline $\mathrm{V}_{5}$ & $6.09 \mathrm{ab}$ & $0.22 \mathrm{a}$ & $28.11 \mathrm{~b}$ & $168.81 \mathrm{a}$ & $9.01 \mathrm{bc}$ & $16.37 \mathrm{~d}$ & $35.99 \mathrm{e}$ & $6.25 \mathrm{~d}$ & $3.12 \mathrm{~d}$ & $22.42 \mathrm{~b}$ \\
\hline $\mathrm{V}_{6}$ & $6.38 \mathrm{ab}$ & $0.38 \mathrm{a}$ & $26.06 \mathrm{bc}$ & $167.29 \mathrm{a}$ & $7.87 \mathrm{~d}$ & $16.87 \mathrm{~d}$ & $43.50 \mathrm{c}$ & $7.61 \mathrm{bc}$ & $3.81 \mathrm{bc}$ & $27.08 \mathrm{a}$ \\
\hline \multicolumn{11}{|c|}{ Sowing time (S) } \\
\hline $\mathrm{V}_{1}$ & $7.46 \mathrm{a}$ & $0.38 \mathrm{a}$ & $28.80 \mathrm{a}$ & $214.73 \mathrm{a}$ & $9.25 \mathrm{a}$ & $20.09 \mathrm{a}$ & $45.65 \mathrm{a}$ & $9.81 \mathrm{a}$ & $4.90 \mathrm{a}$ & $28.81 \mathrm{a}$ \\
\hline $\mathrm{V}_{2}$ & $6.54 \mathrm{~b}$ & $0.42 \mathrm{a}$ & $24.97 \mathrm{~b}$ & $162.25 \mathrm{~b}$ & $8.78 \mathrm{a}$ & $17.28 \mathrm{~b}$ & $44.65 \mathrm{~b}$ & $7.45 \mathrm{~b}$ & $3.73 \mathrm{~b}$ & $25.77 \mathrm{ab}$ \\
\hline $\mathrm{V}_{3}$ & $5.73 \mathrm{c}$ & $0.21 \mathrm{a}$ & $28.29 \mathrm{a}$ & $159.07 \mathrm{~b}$ & $9.16 \mathrm{a}$ & $17.62 \mathrm{~b}$ & $43.71 \mathrm{c}$ & $7.25 \mathrm{~b}$ & $3.63 \mathrm{~b}$ & $25.42 \mathrm{ab}$ \\
\hline $\begin{array}{l}\mathrm{V}_{4} \\
(\mathrm{~V} \times \mathrm{S})\end{array}$ & $4.52 \mathrm{~d}$ & $0.27 \mathrm{a}$ & $29.35 \mathrm{a}$ & $131.51 \mathrm{c}$ & $9.15 \mathrm{a}$ & $17.39 \mathrm{~b}$ & $42.46 \mathrm{~d}$ & $6.03 \mathrm{c}$ & $3.01 \mathrm{c}$ & $22.40 \mathrm{~b}$ \\
\hline $\mathrm{V}_{1} \mathrm{~S}_{1}$ & $7.63 \mathrm{a}-\mathrm{c}$ & $0.63 \mathrm{ab}$ & $27.09 \mathrm{~d}-\mathrm{h}$ & $206.36 \mathrm{bc}$ & $9.02 \mathrm{e}-\mathrm{i}$ & $19.01 \mathrm{~b}-\mathrm{e}$ & $46.28 \mathrm{f}$ & $9.67 \mathrm{~b}-\mathrm{d}$ & $4.83 \mathrm{~b}-\mathrm{d}$ & $29.41 \mathrm{a}-\mathrm{c}$ \\
\hline $\mathrm{V}_{2} \mathrm{~S}_{1}$ & $6.75 \mathrm{e}-\mathrm{f}$ & $0.13 \mathrm{c}$ & $29.94 \mathrm{bc}$ & $202.21 \mathrm{bc}$ & $10.36 \mathrm{ab}$ & $20.48 \mathrm{~b}$ & $50.19 \mathrm{~b}$ & $10.23 \mathrm{~b}$ & $5.12 \mathrm{~b}$ & 28.69 a-d \\
\hline $\mathrm{V}_{3} \mathrm{~S}_{1}$ & $8.13 \mathrm{a}$ & $0.25 \mathrm{bc}$ & $29.20 \mathrm{~b}-\mathrm{e}$ & $236.79 \mathrm{a}$ & $10.00 \mathrm{bc}$ & $24.17 \mathrm{a}$ & $50.64 \mathrm{a}$ & $11.99 \mathrm{a}$ & $6.00 \mathrm{a}$ & $30.92 \mathrm{a}$ \\
\hline $\mathrm{V}_{4} \mathrm{~S}_{1}$ & $7.38 \mathrm{~cd}$ & $0.38 \mathrm{bc}$ & $30.06 \mathrm{~b}$ & $221.50 \mathrm{ab}$ & $8.87 \mathrm{f}-\mathrm{i}$ & $20.38 \mathrm{~b}$ & $43.57 \mathrm{k}$ & $9.73 \mathrm{~b}-\mathrm{d}$ & $4.87 \mathrm{~b}-\mathrm{d}$ & $28.78 \mathrm{a}-\mathrm{d}$ \\
\hline $\mathrm{V}_{5} \mathrm{~S}_{1}$ & $8.00 \mathrm{ab}$ & $0.25 \mathrm{bc}$ & $28.63 \mathrm{~b}-\mathrm{f}$ & $229.27 \mathrm{a}$ & $9.12 \mathrm{~d}-\mathrm{g}$ & $18.51 \mathrm{c}-\mathrm{g}$ & $37.64 \mathrm{q}$ & $8.49 \mathrm{e}$ & $4.24 \mathrm{e}$ & $25.93 \mathrm{c}-\mathrm{h}$ \\
\hline $\mathrm{V}_{6} \mathrm{~S}_{1}$ & $6.88 \mathrm{~d}-\mathrm{f}$ & $0.63 \mathrm{ab}$ & $27.90 \mathrm{~b}-\mathrm{f}$ & $192.26 \mathrm{~cd}$ & $8.14 \mathrm{jk}$ & $18.02 \mathrm{~d}-\mathrm{h}$ & $45.60 \mathrm{~g}$ & $8.75 \mathrm{e}$ & $4.37 \mathrm{e}$ & $29.14 \mathrm{a}-\mathrm{c}$ \\
\hline $\mathrm{V}_{1} \mathrm{~S}_{2}$ & $6.88 \mathrm{~d}-\mathrm{f}$ & $1.00 \mathrm{a}$ & $21.34 \mathrm{k}$ & $146.25 \mathrm{~g}-\mathrm{k}$ & $8.53 \mathrm{ij}$ & $15.92 \mathrm{i}-\mathrm{k}$ & $45.36 \mathrm{~h}$ & $6.85 \mathrm{f}-\mathrm{h}$ & $3.43 \mathrm{f}-\mathrm{h}$ & $24.07 \mathrm{e}-\mathrm{i}$ \\
\hline $\mathrm{V}_{2} \mathrm{~S}_{2}$ & $6.00 \mathrm{gh}$ & $0.25 \mathrm{bc}$ & 29.45 b-d & 176.14 ef & $10.64 \mathrm{a}$ & $18.28 \mathrm{~d}-\mathrm{g}$ & $49.43 \mathrm{c}$ & $8.91 \mathrm{de}$ & $4.46 \mathrm{de}$ & 27.71 a-e \\
\hline $\mathrm{V}_{3} \mathrm{~S}_{2}$ & $7.25 \mathrm{c}-\mathrm{e}$ & $0.13 \mathrm{c}$ & $24.96 \mathrm{~g}-\mathrm{i}$ & $180.35 \mathrm{de}$ & $8.96 \mathrm{e}-\mathrm{i}$ & $20.01 \mathrm{bc}$ & $49.31 \mathrm{c}$ & $9.13 \mathrm{c}-\mathrm{e}$ & $4.57 \mathrm{c}-\mathrm{e}$ & $29.91 \mathrm{ab}$ \\
\hline $\mathrm{V}_{4} \mathrm{~S}_{2}$ & $5.88 \mathrm{~h}$ & $0.50 \mathrm{bc}$ & $27.39 \mathrm{c}-\mathrm{g}$ & $160.79 \mathrm{fg}$ & $8.97 \mathrm{e}-\mathrm{i}$ & $18.60 \mathrm{c}-\mathrm{f}$ & 42.691 & $7.07 \mathrm{fg}$ & $3.53 \mathrm{f}$ & $24.11 \mathrm{e}-\mathrm{i}$ \\
\hline $\mathrm{V}_{5} \mathrm{~S}_{2}$ & $6.50 \mathrm{fg}$ & $0.38 \mathrm{bc}$ & $24.50 \mathrm{~h}-\mathrm{j}$ & $159.38 \mathrm{f}-\mathrm{h}$ & $8.65 \mathrm{~g}-\mathrm{j}$ & $15.01 \mathrm{k}$ & $36.54 \mathrm{r}$ & $5.90 \mathrm{i}$ & $2.95 \mathrm{i}$ & $22.40 \mathrm{~g}-\mathrm{i}$ \\
\hline $\mathrm{V}_{6} \mathrm{~S}_{2}$ & 6.75 e-f & $0.25 \mathrm{bc}$ & $22.20 \mathrm{jk}$ & $150.62 \mathrm{~g}-\mathrm{j}$ & 6.921 & $15.82 \mathrm{i}-\mathrm{k}$ & $44.54 \mathrm{j}$ & $6.85 \mathrm{f}-\mathrm{h}$ & $3.43 \mathrm{f}-\mathrm{h}$ & $26.42 b-f$ \\
\hline $\mathrm{V}_{1} \mathrm{~S}_{3}$ & $6.63 \mathrm{f}$ & $0.25 \mathrm{bc}$ & $22.84 \mathrm{i}-\mathrm{k}$ & $151.39 \mathrm{~g}-\mathrm{i}$ & $8.52 \mathrm{ij}$ & $16.36 \mathrm{~h}-\mathrm{k}$ & $45.08 \mathrm{i}$ & $7.09 \mathrm{f}$ & $3.55 \mathrm{f}$ & $26.17 \mathrm{~b}-\mathrm{g}$ \\
\hline $\mathrm{V}_{2} \mathrm{~S}_{3}$ & $4.13 \mathrm{~lm}$ & $0.25 \mathrm{bc}$ & $35.29 \mathrm{a}$ & $145.38 \mathrm{~g}-\mathrm{k}$ & $10.26 \mathrm{ab}$ & $19.18 \mathrm{~b}-\mathrm{d}$ & $48.75 \mathrm{~d}$ & $7.36 \mathrm{f}$ & $3.68 \mathrm{f}$ & $24.54 \mathrm{e}-\mathrm{i}$ \\
\hline $\mathrm{V}_{3} \mathrm{~S}_{3}$ & $4.88 \mathrm{i}-\mathrm{k}$ & $0.13 \mathrm{c}$ & 29.27 b-e & $140.87 \mathrm{~g}-\mathrm{k}$ & $9.43 \mathrm{de}$ & $19.63 \mathrm{~b}-\mathrm{d}$ & $48.63 \mathrm{~d}$ & $7.13 \mathrm{f}$ & $3.57 \mathrm{f}$ & $24.93 \mathrm{~d}-\mathrm{i}$ \\
\hline $\mathrm{V}_{4} \mathrm{~S}_{3}$ & $5.25 \mathrm{i}$ & $0.25 \mathrm{bc}$ & $26.75 \mathrm{e}-\mathrm{h}$ & 140.38 h-k & $8.97 \mathrm{e}-\mathrm{i}$ & $17.33 \mathrm{e}-\mathrm{i}$ & $41.82 n$ & $6.17 \mathrm{~g}-\mathrm{i}$ & $3.08 \mathrm{~g}-\mathrm{i}$ & $22.25 \mathrm{hi}$ \\
\hline $\mathrm{V}_{5} \mathrm{~S}_{3}$ & $6.00 \mathrm{gh}$ & $0.00 \mathrm{c}$ & $26.50 \mathrm{f}-\mathrm{h}$ & $159.13 \mathrm{f}-\mathrm{h}$ & $9.19 \mathrm{~d}-\mathrm{f}$ & $15.05 \mathrm{k}$ & $35.53 \mathrm{~s}$ & $5.89 \mathrm{i}$ & $2.94 \mathrm{i}$ & $23.42 \mathrm{f}-\mathrm{i}$ \\
\hline $\mathrm{V}_{6} \mathrm{~S}_{3}$ & $7.50 \mathrm{bc}$ & $0.38 \mathrm{bc}$ & 29.07 b-f & $217.29 \mathrm{ab}$ & 8.60 h-j & $18.18 \mathrm{~d}-\mathrm{g}$ & $42.45 \mathrm{~m}$ & $9.88 \mathrm{bc}$ & $4.94 \mathrm{bc}$ & $31.23 \mathrm{a}$ \\
\hline $\mathrm{V}_{1} \mathrm{~S}_{4}$ & $5.25 \mathrm{i}$ & $0.25 \mathrm{bc}$ & $24.97 \mathrm{~g}-\mathrm{i}$ & $130.91 \mathrm{jk}$ & $9.16 \mathrm{~d}-\mathrm{g}$ & $17.13 \mathrm{f}-\mathrm{j}$ & $43.60 \mathrm{k}$ & $6.13 \mathrm{hi}$ & $3.07 \mathrm{hi}$ & $21.93 \mathrm{i}$ \\
\hline $\mathrm{V}_{2} \mathrm{~S}_{4}$ & $4.13 \mathrm{~lm}$ & $0.25 \mathrm{bc}$ & $33.66 \mathrm{a}$ & $138.77 \mathrm{i}-\mathrm{k}$ & $10.23 \mathrm{ab}$ & $18.53 \mathrm{c}-\mathrm{g}$ & $47.26 \mathrm{e}$ & $7.02 \mathrm{f}-\mathrm{h}$ & $3.51 \mathrm{fg}$ & $24.99 \mathrm{~d}-\mathrm{i}$ \\
\hline $\mathrm{V}_{3} \mathrm{~S}_{4}$ & $4.50 \mathrm{j}-1$ & $0.25 \mathrm{bc}$ & $29.69 \mathrm{bc}$ & $133.15 \mathrm{i}-\mathrm{k}$ & $9.59 \mathrm{~cd}$ & $18.98 \mathrm{~b}-\mathrm{e}$ & $47.60 \mathrm{~d}$ & $6.74 \mathrm{f}-\mathrm{i}$ & $3.37 \mathrm{e}$ & $24.15 \mathrm{e}-\mathrm{i}$ \\
\hline $\mathrm{V}_{4} \mathrm{~S}_{4}$ & $5.00 \mathrm{ij}$ & $0.38 \mathrm{bc}$ & $29.94 \mathrm{bc}$ & $149.81 \mathrm{~g}-\mathrm{j}$ & 8.99 e-i & $17.34 \mathrm{e}-\mathrm{i}$ & $40.62 \mathrm{p}$ & $6.58 \mathrm{f}-\mathrm{i}$ & $3.29 \mathrm{f}-\mathrm{i}$ & $23.89 \mathrm{e}-\mathrm{i}$ \\
\hline $\mathrm{V}_{5} \mathrm{~S}_{4}$ & $3.88 \mathrm{~m}$ & $0.25 \mathrm{bc}$ & $32.79 \mathrm{a}$ & $127.45 \mathrm{kl}$ & $9.10 \mathrm{~d}-\mathrm{h}$ & $16.89 \mathrm{~g}-\mathrm{j}$ & $34.27 \mathrm{t}$ & $4.72 \mathrm{j}$ & $2.36 \mathrm{j}$ & $17.93 \mathrm{j}$ \\
\hline $\mathrm{V}_{6} \mathrm{~S}_{4}$ & $4.38 \mathrm{k}-\mathrm{m}$ & $0.25 \mathrm{bc}$ & $25.06 \mathrm{~g}-\mathrm{i}$ & 109.001 & $7.82 \mathrm{k}$ & $15.46 \mathrm{jk}$ & $41.39 \mathrm{o}$ & $4.96 \mathrm{j}$ & $2.48 \mathrm{j}$ & $21.53 \mathrm{ij}$ \\
\hline $\mathrm{CV}(\%)$ & 13.18 & 16.67 & 15.05 & 14.06 & 10.61 & 14.29 & 10.47 & 6.02 & 6.01 & 9.31 \\
\hline $\begin{array}{l}\text { LSD } \\
(0.05)\end{array}$ & 0.59 & 0.47 & 2.59 & 20.23 & 0.51 & 1.69 & 0.20 & 0.90 & 0.43 & 3.90 \\
\hline
\end{tabular}

Mean in a vertical column followed by same letter or without letter do not differ significantly at $5 \%$ level. 
In case of combined treatments, findings revealed that the maximum number of effective tillers per plant was recorded from BARI Gom-25 when sown on November $15\left(\mathrm{~V}_{3} \mathrm{~S}_{1}\right)$ which was statistically identical to $\mathrm{V}_{1} \mathrm{~S}_{1}$ and $\mathrm{V}_{5} \mathrm{~S}_{1}$ treatments (Table 5). This finding is contradictory to the results of Shahzad et al. (2007) who reported that wheat sown on December produced significantly more number of productive tillers than the crop sown on November. The highest number of grains (236.79) per plant was also recorded from $\mathrm{V}_{3} \mathrm{~S}_{1}$ treatment which was significantly different from majority of the treatments. However, the maximum number of grains per spike was obtained from $\mathrm{V}_{2} \mathrm{~S}_{3}$ treatment. Jahan and Adam (2015) also reported nonsignificantly higher number of grains per plant in BARI Gom-25 sown on November 24. Besides results showed that the maximum length of spike was obtained from BARI Gom-24 when sown on November $22\left(\mathrm{~V}_{2} \mathrm{~S}_{2}\right)$ followed by November $15\left(\mathrm{~V}_{2} \mathrm{~S}_{1}\right)$. Research report revealed that earlier planting resulted in better spike development due to longer growing period (Waraich et al.1981).

Results of this study also showed that significantly maximum dry weight of spike, 1000-grain weight, yield per plant and yield per hectare were recorded from BARI Gom-25 when sown on November 15. Findings of Jahan and Adam (2015) revealed that November 14 sown wheat produced maximum 1000-grain weight and there was a gradual decreasing trend with delayed sowing. The results are also in agreement with the previous reports (Subhan et al. 2004, Abdullah et al. 2007, Shahzad et al. 2007).

Table 5 shows that sowing time had significant effect on yield per plant and yield per hectare and findings of this experiment indicated that November 15 was the most suitable sowing time for most of the varieties. Results also indicated that yield of wheat varieties decreased gradually with delayed sowing although, BARI Gom-28 produced higher yield on November 29 than other sowing time. Shah and Akmal (2002) and Ali et al. (2010) reported that different varieties responded differently on different sowing dates. Findings of present investigation also showed that BARI Gom-25 $\left(\mathrm{V}_{3}\right)$ produced maximum yield $(11.99 \mathrm{~g})$ on optimum time of sowing followed by BARI Gom-24 (10.23 g). Yield of BARI Gom-25 reduced by 23.85, 40.53 and $43.79 \%$ at 7, 14 and 21 days delay in sowing, respectively. Jahan and Adam (2015) obtained statistically similar yield from November 14 and November 24 sown BARI Gom-25 but decreased significantly beyond the time of November 24. Decrease in grain yield of wheat due to delay sowing from November 20 onward was also reported by Singh and Uttam (1999). Ansary et al. (1989) and Shahzad et al. (2007) also obtained lower yield with delay sown wheat. Regardless of varieties or cultivars, better yields were obtained when wheat was sown after November 15 and before November 30 (Akhtar et al. 2006). Baloch et al. (2010) recorded maximum grain yield when sown in November while minimum in December. Among the six varieties, BARI Gom-25 produced maximum harvest index (27.48). Results also revealed that seeds sown on November 15 resulted the highest $\mathrm{HI}$ (28.81). In case of combined effect, although $\mathrm{HI}$ was recorded maximum due to BARI Gom-28 sown in November $29\left(\mathrm{~V}_{6} \mathrm{~S}_{3}\right)$ followed by BARI Gom-25 sown in November 15 $\left(\mathrm{V}_{3} \mathrm{~S}_{1}\right)$, but both of them were not found statistically different.

Out of six varieties of wheat, BARI Gom-25 was the best performed variety and among the four sowing time, November 15 was optimum for most of the varieties.

\section{References}

Abdullah M, Rehman A, Ahmad N and Rasul I 2007. Planting time effect on grain and quality characteristics of wheat. Pakistan J. Agril. Sci. 44(2): 200-202.

Akhtar M, Cheema MS, Jamil M and Ali L 2006. Effect of time of sowing on some important characters of wheat (Triticum aestivum) genotypes. J. Agric. Res. 44(4): 255-259.

Ali MA, Ali M, Sattar M and Ali L 2010. Sowing date effect on yield of different wheat varieties. J. Agric. Res. 48(2): 157-162. 
Ansary AH, Khushak AM, Sethar MA, Ariam NA and Emon MYM 1989. Effect of sowing dates on growth and yield of wheat cultivars. Pakistan J. Sci. Ind. Res. 32: 39-42.

Azad AK, Rahman L, Gosami BK, Malakar PK, Hasan S, Barma NCD, Pal TK, Aziz A, Hossen S, Alam SN, Hasaan K, Rahman B and Rahman HH 2007. Krishi Projukti Manual (Agro-technology Manual). Bangladesh Agricultural Research Institute, Gazipur 1701, Bangladesh. pp. 292.

Baloch MS, Shah ITH, Nadim MA, Khan MI and Khakwani AA 2010. Effect of seeding density and planting time on growth and yield attributes of wheat. J. Anim. Plant Sci. 20(4): 239-242.

Chowdhury MAH and Hasan MS 2013. Handbook of agricultural technology. Bangladesh agricultural technology. Bangladesh Agric. Res. Council, Farmgate, Dhaka. pp. 230.

Dabre WM, Lall SB and Lngole GL 1993. Effects of sowing dates on yield, ear number, stomatal frequency and stomatal index in wheat. J. Maharashatra Agril. Univ.18: 64-66.

Fertilizer Recommendation Guide 2012. Bangladesh Agricultural Research Council, Farmgate, Dhaka-1215. pp. 274.

Hobbs PR and Gupta RK 2002. Resource conserving technologies for wheat in rice-wheat systems. In: Ladha JK, James EH, Duxbury JD, Gupta RK and Buresh RJ (eds.) Improving the productivity and sustainability of rice-wheat systems: Issues and impact. ASA Special Publication, ASA, Madison, Wisconsin, USA.

Jackson ML 1973. Soil Chemical Analysis. Asia Pub. House, Bombey, Calcutta, New Delhi, Madras. pp. 151-154.

Jahan N and Adam AMMG 2015. Growth and yield responses of BARI Gom-25 in relation to sowing time. Dhaka Univ. J. Biol. Sci. 24(1): 99-102.

Marr IL and Cresser MS 1983. Environmental chemical analysis. University of California. International Textbook Co. 258 pp.

Murphy J and Riley JP 1962. A modified simple solution method for determination of phosphate in saturated water. Analytica. Chemica. Acta. 27: 31-36.

Rahman MA, Hossain SJ, Hossain MB, Amin MR and Sarkar KK 2010. Effect of variety and culture method on the yield and yield attributes of wheat. Int. J. Sustain. Crop Prod. 5(3):17-21.

Rashid MH, Samanta SC, Biswas P, Mannan MA and Zaman AKMM 2004. Performance of thirty wheat genotypes under late sown condition in sourthern region of Bangladesh. Asian J. Pl. Sci. 3(3): 286-289.

Rosegrant MW and Agcaoili M 2010. Global food demand, supply and price prospects to 2010. Int. Food Policy Res. Inst. Washington, DC, USA.

Saifuzzaman M, Barma NCD, Hossain ABS, Rawson HM, Kabir J, Rahman MM and Dalgliesh NP 2010. How to grow wheat in southern Bangladesh and fit it into a timely annual sequence with other crops (1st ed). Wheat research centre, BARI, Gazipur 1701, Bangladesh. p. 52.

Shah SSM and Akmal M 2002. Effect of different sowing dates on yield and yield components of wheat varieties. Sarhad J. Agric. 18(2): 143-149.

Shahzad MA, Din WU, Sahi ST, Khan MM, Ehsanullah and Ahmad M 2007. Effect of sowing dates and seed treatment on grain yield and quality of wheat. Pakistan J. Agri. Sci. 44(4): 581-583.

Singh VPN and Uttam SK 1999. Influence of sowing dates on yield of wheat cultivars under saline sodic conditions in Central Utter Pradesh. Indian J. Agric. 38: 64-68.

Steel RGD, Torrie JH and Dickey DA 1997. Principles and procedures of statistics. McGraw Hill Book Co. Inc. New York. pp. 666.

Subhan F, Khan M and Jamro GH 2004. Effect of different planting date, seeding rate and weed control method on grain yield and yields components in wheat. Sarhad J. Agri. 20(1): 51-55.

Waraich SA, Yasmin S and Ashraf S 1981. Genetic parameters influenced by seeding dates in wheat. Pakistan J. Agric. Res. 3: 273-276. 